\title{
Efectividad y tolerabilidad del ácido zoledrónico en el tratamiento del cancer de próstata metastásico
}

Cózar Olmo $\mathrm{JM}^{1}$, Carballido Rodríguez J², Luque Galvez $\mathrm{P}^{3}$, Tabernero Gómez $\mathrm{AG}^{4}$, Barreiro Mouro A ${ }^{5}$, Sánchez Sánchez E ${ }^{6}$, González Enguita $C^{7}$, Alcover García J33 , García-Galisteo E ${ }^{8}$, Abascal García JM99, Sánchez Chapado $\mathrm{M}^{10}$, GómezVeiga $\mathrm{F}^{11}$, Minguez Martínez RJ ${ }^{12}$, Rodríguez Antolín A ${ }^{13}$, Medina López RA ${ }^{14}$, Rico Morales $\mathrm{M}^{15}$, Regadera Sejas FJ ${ }^{16}$, Búcar Terrades $\mathrm{S}^{17}$, Jamardo González D ${ }^{18}$, Gálvez $\mathrm{R}^{19}$.

${ }^{1}$ Servicio de Urología H. Universitario Virgen de las Nieves, Granada; ${ }^{2}$ Unidad de Urología H. Puerta de Hierro, Madrid; ${ }^{3}$ Servicio de Urología H. Clínico Provincial de Barcelona; ${ }^{4}$ Unidad de Urología H. La Paz, Madrid; ${ }^{5}$ Servicio de Urología Complejo Hospitalario de Ourense (H. Cristal), Ourense; ${ }^{6}$ Servicio de Urología H. Virgen de la Macarena, Sevilla; ${ }^{7}$ Servicio de Urología Fundación Jiménez Díaz, Madrid; ${ }^{8}$ Servicio de Urología H. Regional Carlos Haya, Málaga; ${ }^{9}$ Servicio de Urología H. Central de Asturias, Oviedo; ${ }^{10}$ Servicio de Urología H. Príncipe de Asturias, Alcalá de Henares; ${ }^{11}$ Servicio de Urología H. Juan Canalejo, A Coruña; ${ }^{12}$ Servicio de Urología H. La Princesa, Madrid; ${ }^{13}$ Servicio de Urología H. Universitario 12 de Octubre, Madrid; ${ }^{14}$ Servicio de Urología H. Virgen del Rocío, Sevilla; ${ }^{15}$ Servicio de Urología H. Xeral-Calde, Lugo; ${ }^{16}$ Servicio de Urología H. Central de Asturias, Oviedo; ${ }^{17}$ Servicio de Urología H. Municipal de Badalona; ${ }^{18}$ Servicio de Urología H. Meixoeiro de Vigo; ${ }^{19}$ Servicio de Unidad del Dolor H. Universitario Virgen de las Nieves, Granada.

Actas Urol Esp. 2008;32(5):492-501

\section{RESUMEN}

EFECTIVIDAD Y TOLERABILIDAD DEL ÁCIDO ZOLEDRÓNICO EN EL TRATAMIENTO DEL CANCER DE PRÓSTATA METASTÁSICO Objetivos: Evaluar la efectividad y tolerabilidad del ácido zoledrónico en pacientes con cáncer de próstata y metástasis óseas en fase hormonosensible (HS) y hormonoindependiente (HI).

Material y Métodos: Se diseñó un estudio de ámbito nacional, observacional, prospectivo, abierto, y multicéntrico, Se incluyeron un total de 218 varones diagnosticados de cáncer de próstata en fase HS (36\%) o HI (64\%) que recibieron, además del tratamiento oncológico específico, ácido zoledrónico ( $4 \mathrm{mg} / \mathrm{IV} / \mathrm{mes}$ durante 6 meses).

Se evaluó la efectividad mediante: 1) Evaluación de la mejoría del dolor y movilidad; 2) Incidencia y tiempo de aparición de eventos esqueléticos (TEE); y 3) Análisis de marcadores óseos.

La tolerabilidad se estudió registrando el número y tipo de efectos adversos. Se realizó una encuesta de satisfacción al paciente tras finalizar el tratamiento.

Resultados: De los 218 pacientes, 170 (78\%) fueron evaluables para efectividad. En todos ellos, ya fueran del grupo HS o HI, se observó una disminución de la puntuación del dolor en reposo y en movimiento ( $\mathrm{p}<0,0001$ ), una mejora en la movilidad ( $\mathrm{p}=0,005)$, y en la calidad de vida.

La incidencia global de eventos esqueléticos fue del $11,2 \%$, con un TEE de 10,7 meses. No hubo diferencias significativas entre los pacientes HS respecto a los HI. Los marcadores de osteolisis (N-telopéptido) descendieron significativamente con el tratamiento, tanto en los HS como HI.

Para seguridad fueron evaluables 212 pacientes (97,2\%). La incidencia de las reacciones adversas fue del 16\% (34/212), siendo significativamente mayor en los pacientes HS $(22,4 \%)$ con respecto a los HI (11,9\%). Globalmente la tolerabilidad al ácido zoledrónico fue buena, sin morbilidad significativa entre ambos grupos (HS y $\mathrm{HI}$ )

Un $66 \%$ de los pacientes contestaron sentirse satisfechos o muy satisfechos.

Conclusiones: El ácido zoledrónico se mostró eficaz para aliviar el dolor, mejorar la movilidad y aumentar la calidad de vida y reducir o retrasar los eventos esqueléticos en los pacientes con cáncer de próstata con enfermedad ósea metastásica sintomática, independientemente de la fase, HS o HI en que se encuentren. La tolerabilidad y la satisfacción de los pacientes fue buena.

Palabras clave: Cáncer de próstata metastásico. Ácido Zoledrónico. Eventos óseos. Calidad de vida.

\section{ABSTRACT}

EFFECTIVENESS AND TOLERABILITY OF ZOLEDRONIC ACID IN THE TREATMENT OF METASTATIC PROSTATE CANCER

Objetives: To assess the effectiveness and tolerability of zoledronic acid in prostate cancer patients with bone metastases at the hormone-sensitive (HS) and hormone-independent (HI) stages.

Materials and Methods: A nationwide, observational, prospective, open and multi-centre trial was devised, with a total of 218 male patients diagnosed with prostate cancer at the HS stage (36\%) or HI stage $(64 \%)$ who were administered zoledronic acid (4 mg/IV/month for 6 months) in addition to their specific oncological treatment.

Effectiveness was assessed by the following means: 1) Assessment of the improvement in pain and mobility; 2) Incidence and time to onset of skeletal-related events (SREs) and 3) Analysis of bone markers.

Tolerability was assessed by means of registering the number and type of adverse effects. A satisfaction survey was carried out amongst the patients after the end of the trial.

Results: Out of the 218 patients, $170(78 \%)$ were evaluable for effectiveness. A decrease in pain ratings at rest and during movement was observed in all patients, whether in the HS or HI groups ( $<<0,0001)$. Improved mobility was observed likewise ( $p=0,005)$, as was quality of life.

The global incidence of skeletal events was $11.2 \%$, with a time to onset of SREs of 10.7 months. There were no significant differences observed between HS vs. HI patients.

Osteolysis markers (N-telopeptide) decreased significantly with the treatment across both the HS and HI groups.

For safety reasons, 212 patients were evaluable (97.2\%). The incidence of adverse drug reactions was $16 \%(34 / 212)$ and was found to be significantly higher in HS patients (22.4\%) compared with HI patients (11.9\%). Overall, the tolerability of zoledronic acid was good, with no significant morbidity in either group (HS and $\mathrm{HI}) .66 \%$ of the patients reported feeling satisfied or very satisfied.

Conclusions: Zoledronic acid proved effective in the relief of pain, improving mobility and quality of life as well as reducing or delaying the occurrence of skeletal-related events in prostate cancer patients presenting metastatic bone disease, regardless of the phase, whether HS or HI, they found themselves in. Tolerability and patient satisfaction were rates as good.

Keywords: Metastatic prostate cancer. Zoledronic acid. Skeletal events. Quality of life. 
$\mathrm{L}^{\mathrm{o}}$ os ensayos clínicos han demostrado que el ácido zoledrónico disminuye la incidencia de complicaciones óseas en pacientes con mieloma múltiple y en pacientes con metástasis secundarias a tumores sólidos, entre ellos, el cáncer de próstata ${ }^{1-6}$.

Saad et al. ${ }^{7}$ evaluaron el ácido zoledrónico en 600 pacientes en fase Hormonoindependiente (HI) y observaron que existía una reducción significativa en el número de eventos óseos, complicaciones esqueléticas (fracturas patológicas vertebrales y no vertebrales) con un retraso de más de 5 meses en los que finalmente la presentaban, y un alivio del dolor óseo. Todo ello con una mínima toxicidad sin diferencias significativas respecto al placebo. En este importante estudio, no se incorporaron pacientes con cáncer de próstata metastático en estadio hormonosensible (HS). Sus criterios de inclusión sólo aceptaban pacientes en progresión hormonoindependiente (HI). Existiendo controversia entre los distintos autores y foros de discusión, de si tales resultados deben ser o no extrapolados a los pacientes en fase metastásica $\mathrm{HS}^{8}$, o si por el contrario, en estos no tenemos justificación suficiente como para indicar el uso del ácido zoledrónico ${ }^{9}$.

Para responder este interrogante se diseñó un estudio multicéntrico, prospectivo, observacional, y abierto, para evaluar la efectividad del ácido zoledrónico en pacientes diagnosticados de cáncer de próstata con enfermedad ósea metastásica, bien en estadio HS (estadio D-2 de Whitmore-Jewett) o en fase de progresión HI.

Los objetivos del estudio fueron: mejorar el manejo del dolor y movilidad; disminuir o retrasar la aparición de efectos adversos severos; mejorar el estado funcional y la calidad de vida de los pacientes; y evaluar la seguridad del tratamiento.

\section{MATERIAL Y MÉTODOS}

La población en estudio fue de pacientes mayores de 18 años con diagnóstico histopatológico de carcinoma de próstata. Se les debía haber practicado deprivación androgénica, y haber desarrollado metástasis óseas dolorosas (VAS en movimiento $\geq$ 2). Con un estado general razonable (ECOG $\leq 2$ o Índice de Karnofsky $\geq 70$ ).

Todos los pacientes incluidos fueron informados y firmaron el consentimiento informado, asimismo todos los pacientes cumplían todos los criterios de inclusión y ninguno de los de exclusión.
Se les administró ácido zoledrónico (4 mg) en infusión de 15 minutos, cada 4 semanas ( \pm 1 semana). Se asociaron suplementos de calcio y vitamina D (500 mg/d y $400 \mathrm{mg} / \mathrm{d}$, respectivamente) mientras duró la administración del bisfosfonato, siempre que no hubiera hipercalcemia.

Los pacientes que abandonaron el estudio antes del cumplimiento del tratamiento, y para los cuales constó como mínimo una evaluación de su estado posterior al inicio del estudio, debieron ser considerados en el análisis por intención de tratar. Asimismo, todos los pacientes que recibieron al menos una dosis del fármaco en estudio debieron ser incluidos en el análisis de seguridad.

Tratándose de un estudio no controlado, que tiene solo finalidad descriptiva, se previó la participación de 218 pacientes a los que se les administraron 6 dosis del fármaco en estudio.

Con los criterios clínicos y bioquímicos disponibles fue posible clasificar en 2 subgrupos bien caracterizados a todos los pacientes. Aquellos en los que existía sensibilidad en la respuesta al tratamiento hormonal determinada por el comportamiento de los valores de PSA (receso y estabilización) y por la respuesta clínica y antiálgica, a los que clasificamos como pacientes hormonosensibles (HS) $\mathrm{n}=76$. Y los que exhibían refractariedad terapéutica a la manipulación hormonal determinada por los valores del PSA (incremento $>50 \%$ en 3 determinaciones consecutivas), por la mayor demanda de analgésicos y por la necesidad de incorporar otros tratamientos antineoplásicos. Los clasificamos como pacientes hormonoindependientes (HI) $n=135$.

La duración del tratamiento fue de 6 dosis administradas en un intervalo de 18-30 semanas con 8 visitas de seguimiento.

El dolor en reposo y en movimiento se midió en dos visitas mensuales consecutivas de forma cuantitativa mediante la escala EVA (Evaluación Visual Analógica). Se determinó la movilidad del paciente mediante la escala de Latineen. Con el cuestionario SF-36 se evaluó el estado general y la calidad de vida de los pacientes al inicio del estudio y en cada visita de seguimiento. Evento Esquelético (EE) fue definido antes del inicio del estudio como cualquier fractura patológica, vertebral y no vertebral, radiación para el dolor, radiación para el tratamiento de fracturas, o compresión de la medula espinal asociada por compresión vertebral. Se evaluó la incidencia y el tiempo de aparición de los EE. 
El criterio principal en la evaluación de seguridad fue la aparición de acontecimientos adversos, registrándose su tipo y gravedad.

\section{Análisis de datos y pruebas estadísticas}

Las comparaciones de las variables de interés a lo largo de las diferentes visitas fueron llevadas a cabo mediante el test no paramétrico de Wilcoxon (comparación de datos basales con el resto de visitas para dos muestras relacionadas).

La comparación entre subgrupos de la muestra se llevó a cabo mediante el test no paramétrico de Mann-Whitney (dos grupos) o bien mediante el test de Kruskal-Wallis (más de dos grupos).

Las variables cualitativas se analizaron mediante tablas de contingencia (test Chi cuadrado). Todos los tests estadísticos se realizaron mediante un contraste bilateral con un nivel de significación · del 5\%

Se evalúa el tiempo de aparición de Eventos Esqueléticos durante el estudio mediante curvas de Kaplan-Meier.

El análisis estadístico se llevó a cabo mediante el programa Statistical Package Social Science (SPSS, v. 13.0).

\section{RESULTADOS}

Se reclutaron un total de 218 pacientes (36\% en fase HS y $64 \%$ en fase HI), mediante la colaboración de 30 hospitales participantes. La edad media de todos ellos en el momento del diagnóstico de cáncer de próstata fue de 68,6 años $(\mathrm{SD}=7,5)$. El tiempo transcurrido desde el diagnóstico de metástasis óseas y desde el diagnóstico de cáncer hasta el inicio del presente estudio fue superior en los pacientes HI ( 1,1 y 3,3 años respectivamente) que en los pacientes HS (0,6 y 1,1 años respectivamente); ( $<<0,05)$.

Las localizaciones de la metástasis con mayor incidencia entre los pacientes fueron la columna vertebral, la torácico-costal y la pelvis ósea.

El status performance de los pacientes de los grupos HS y HI no presentó diferencias estadísticamente significativas a su inclusión en el estudio (Tablas 1 y 2).

Se consideraron valorables para el análisis de efectividad el 78\% ( $n=170)$ de los casos, quedando fuera del análisis de efectividad 48 casos por violación de los criterios de inclusión/exclusión

Se hallaron diferencias entre los dos grupos de pacientes (HI y HS) respecto a la mejoría del dolor en reposo durante los meses $1,3,4,5$ y 6 de tratamiento $(\mathrm{p}<0,05)$, a favor del grupo HS (Fig. 1). La diferencia de puntuaciones entre basal y seguimiento fue estadísticamente significativa $(\mathrm{p}<0,0001)$

Los pacientes en fase HS iniciaron el tratamiento con una puntuación de dolor en movimiento de $5,3$ puntos ( $\mathrm{SD}=1,9)$, y presentaron un descenso de la puntuación del dolor durante los 6 meses de tratamiento de 1,8 puntos, hasta situarse con una puntuación de 3,5 puntos ( $\mathrm{SD}=2,8)$. Los pacientes en fase HI mostraron durante la visita basal una puntuación del dolor en movimiento de 6,4 puntos $(\mathrm{SD}=2,0)$, descendiendo durante los 6 meses de tratamiento 1,8 puntos, hasta los 4,6 puntos ( $\mathrm{SD}=2,9)$. Si bien existió una mejora del dolor en movimiento

Tabla 1. Datos del Status Performance de los pacientes

\begin{tabular}{|c|c|c|c|c|c|c|c|c|c|c|}
\hline & & $\mathbf{n}^{(1)}$ & Media & SD & Mediana & $\mathbf{P}_{25}$ & $\mathbf{P}_{75}$ & Mín. & Máx. & $\mathbf{P}^{(2)}$ \\
\hline Estadol & Hormonosensibles & 73 & 1,3 & 0,8 & 1,0 & 1,0 & 2,0 & 0,0 & 4,0 & 0,1035 \\
\hline funciona & Hormonoindependientes & 133 & 1,4 & 0,7 & 1,0 & 1,0 & 2,0 & 0,0 & 4,0 & \\
\hline (ECOG) & Global & 207 & 1,4 & 0,8 & 1,0 & 1,0 & 2,0 & 0,0 & 4,0 & - \\
\hline Estado & Hormonosensibles & 74 & 77,8 & 12,2 & 80,0 & 70,0 & 90,0 & 30,0 & 100,0 & 0,1770 \\
\hline funcional & Hormonoindependientes & 133 & 76,5 & 11,4 & 80,0 & 70,0 & 80,0 & 10,0 & 100,0 & \\
\hline (I.K.) & Global & 208 & 77,0 & 11,7 & 80,0 & 70,0 & 80,0 & 10,0 & 100,0 & - \\
\hline Estado & Hormonosensibles & 72 & 33,8 & 10,5 & 32,3 & 26,1 & 41,9 & 13,4 & 56,1 & 0,1559 \\
\hline físico & Hormonoindependientes & 129 & 31,1 & 9,7 & 30,6 & 23,9 & 37,4 & 10,0 & 55,4 & \\
\hline (SF-36) & Global & 202 & 32,2 & 10,1 & 31,4 & 24,6 & 38,8 & 10,0 & 56,1 & - \\
\hline Estado & Hormonosensibles & 72 & 45,3 & 11,4 & 48,7 & 38,0 & 53,7 & 18,4 & 63,7 & 0,6894 \\
\hline mental & Hormonoindependientes & 129 & 44,0 & 13,4 & 46,1 & 32,8 & 54,5 & 7,1 & 70,3 & \\
\hline$(\mathrm{SF}-36)$ & Global & 202 & 44,4 & 12,7 & 47,2 & 34,1 & 54,0 & 7,1 & 70,3 & - \\
\hline
\end{tabular}

(1) En algunos casos no se especificó el dato.

(2) Test U de Mann-Whitney. 
Tabla 2 Estado funcional ECOG e I.K. categorizados

\begin{tabular}{|c|c|c|c|c|c|c|c|}
\hline & & \multicolumn{2}{|c|}{ Global } & \multicolumn{2}{|c|}{ Hormonosensibles } & \multicolumn{2}{|c|}{ Hormonoindependientes } \\
\hline & & $\mathbf{n}$ & $\%(1)$ & $\mathbf{n}$ & $\%(1)$ & $\mathbf{n}$ & $\%(1)$ \\
\hline Estado & Total pacientes ${ }^{(2)}$ & 207 & $100 \%$ & 73 & $100 \%$ & 133 & $100 \%$ \\
\hline funcional & 0 & 16 & $7,7 \%$ & 7 & $9,6 \%$ & 9 & $6,8 \%$ \\
\hline \multirow[t]{3}{*}{$(\mathrm{ECOG})^{(3)}$} & 1 & 114 & $55,1 \%$ & 45 & $61,6 \%$ & 68 & $51,1 \%$ \\
\hline & 2 & 63 & $30,4 \%$ & 15 & $20,5 \%$ & 48 & $36,1 \%$ \\
\hline & $>2$ & 14 & $6,8 \%$ & 6 & $8,2 \%$ & 8 & $6,0 \%$ \\
\hline Estado & Total pacientes $^{(2)}$ & 208 & $100 \%$ & 74 & $100 \%$ & 133 & $100 \%$ \\
\hline funcional $^{(4)}$ & $100 \%-90 \%$ & 48 & $23,1 \%$ & 21 & $28,4 \%$ & 27 & $20,3 \%$ \\
\hline \multirow[t]{3}{*}{ (I.K.) } & $89 \%-80 \%$ & 84 & $40,4 \%$ & 31 & $41,9 \%$ & 52 & $39,1 \%$ \\
\hline & $79 \%-70 \%$ & 52 & $25,0 \%$ & 12 & $16,2 \%$ & 40 & $30,1 \%$ \\
\hline & $<70 \%$ & 24 & $11,5 \%$ & 10 & $13,5 \%$ & 14 & $10,5 \%$ \\
\hline
\end{tabular}

${ }^{(1)}$ Porcentaje calculado sobre el total de pacientes que aportaron el dato

(2) En algunos casos no se especificó el dato.

(3) No se hallaron diferencias estadisticamente significativas entre ambos grupo de pacientes (test Chi-cuadrado, $p=0,1390$ ).

(4) No se hallaron diferencias estadísticamente significativas entre ambos grupo de pacientes (test Chi-cuadrado, p=0,1427).

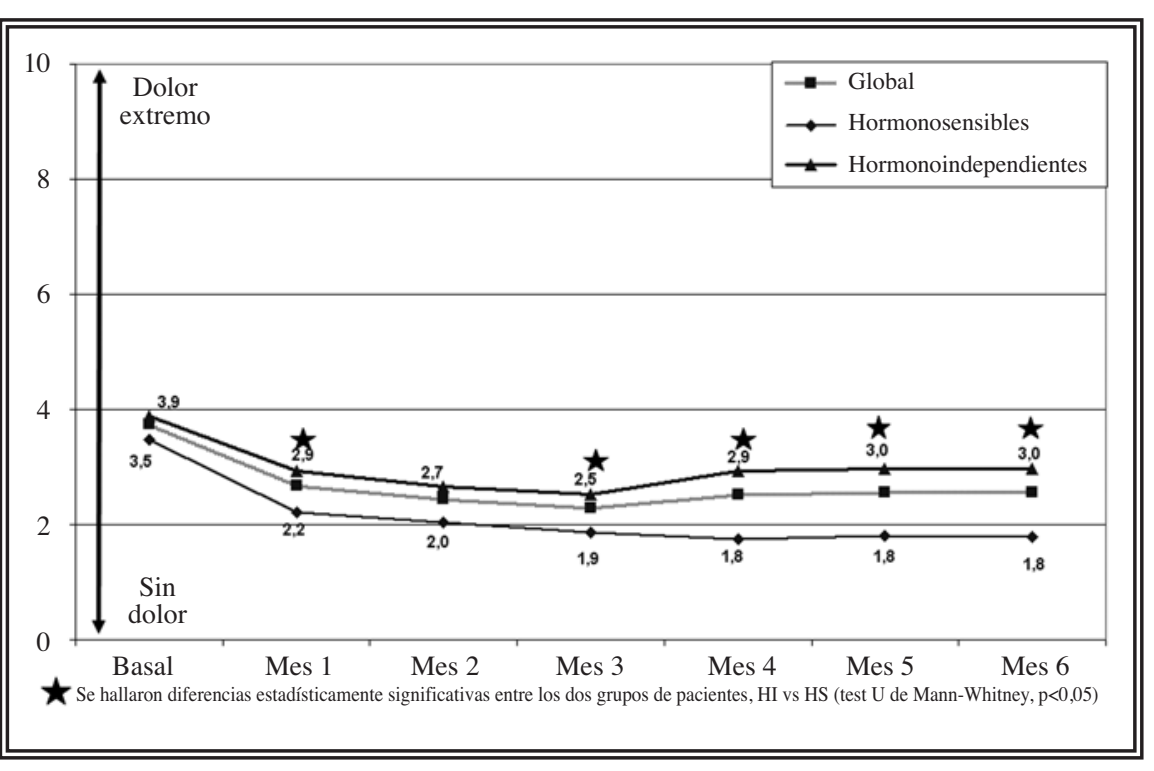

FIGURA 1. Evolución de la puntuación del dolor en reposo (VAS en reposo).
Tanto los pacientes HS como los pacientes $\mathrm{HI}$ mostraron un ligera mejoría $(\mathrm{p}<0,05)$ de su estado físico (Fig. 4).

Respecto al estado mental de los pacientes no se observaron cambios significativos. Se hallaron diferencias estadísticamente significativas entre $\mathrm{HS} \mathrm{y} \mathrm{HI}$ en la visita a los 3 meses en las puntuaciones $(\mathrm{p}<0,05)$ pero no en la visita basal ni a los 6 meses (p>0,05) (Fig. 5).

Del total de 170 pacientes valorables para efectividad, un 11,2\% $(n=19)$ presentó algún Evento Esquelético durante los seis meses de seguimiento, ocurriendo 6 eventos en 6 pacientes HS y 15 eventos en 13 pacientes HI.

No se hallaron diferencias estadísticamente significativas entre la incidencia de eventos óseos en pacientes en fase HI (13 pacientes de 112:11,6\%) y los pacientes en fase HS (6 pacientes de 58: 10,3\%) (test Chi-cuadrado, $\mathrm{p}=0,8044$ ).

Para los pacientes HS el mes con mayor incidencia de eventos fue el segundo mes $(50,0 \%$ de los eventos), mientras que para los pacientes HI fueron por igual el primer, cuarto y quinto mes $(23,1 \%$ de los eventos).

En los pacientes en fase HS el evento esquelético más frecuente fue la fractura patológica, en un $50,0 \%$ de los eventos, y para los pacientes HI el más frecuente fue la radiación para aliviar el dolor, en un 


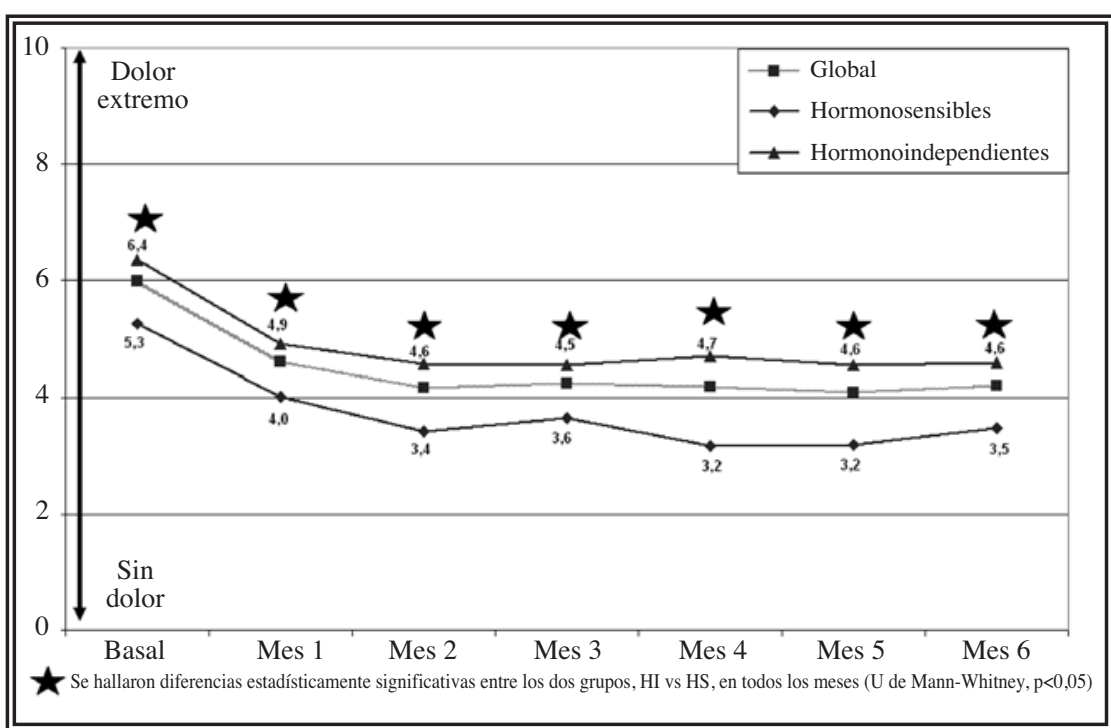

FIGURA 2. Evolución de la puntuación del dolor en movimiento (VAS en movimiento).

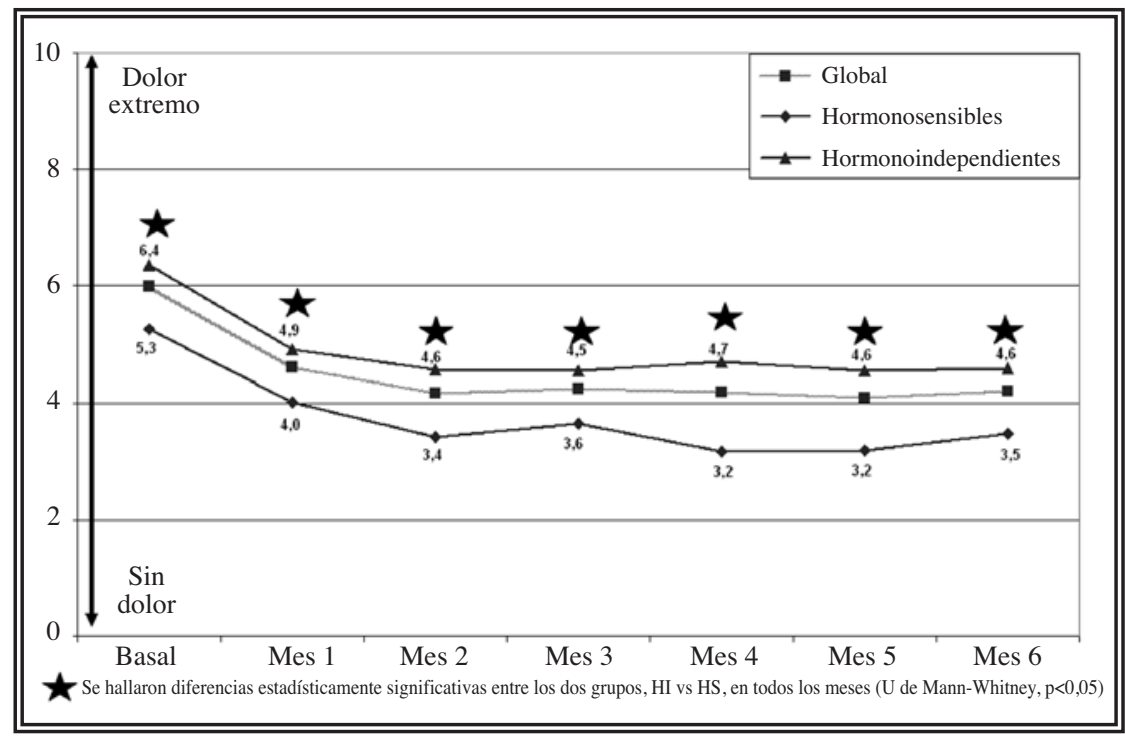

FIGURA 3. Evolución de la puntuación del dolor (indice Lattinen). los pacientes en fase HS. Algo que en la práctica clínica habitual no suele ocurrir, presentando en general los pacientes HI un incremento significativo de eventos óseos con respecto a los pacientes HS.

Al inicio del estudio los pacientes presentaron una fosfata alcalina ósea media de 591,4 $\mathrm{mg} / \mathrm{L}$ $(\mathrm{SD}=1452,7)$, mientras que al sexto mes de seguimiento fue de 329,6 $\mathrm{mg} / \mathrm{dl} \quad(\mathrm{SD}=506,5), \quad$ sin hallarse diferencias estadísticamente significativas entre ambos meses ni para el global de la muestra, ni para los pacientes HS o HI ( $\mathrm{p}>0,05$ ).

Los pacientes mostraron un descenso en el N-telopéptido (NTX) entre la visita basal y el mes 6 de seguimiento, pasando de una NTX de 171,7 nmol $(\mathrm{SD}=204,2)$ al inicio del estudio, a una NTX de 45,2 nmol $(\mathrm{SD}=61,4)$ al final, hallándose diferencias estadísticamente significativas para el global de la muestra, para los pacientes HS y para los HI $(\mathrm{p}<0,05)$ (Tabla 5). Es pues uno de los parámetros analíticos que mejor objetiva la detención de la osteolisis como resultado de la acción del ácido zoledrónico, y cuyos efectos revertirán en la efectividad clinica anteriormente demostrada en las variables estudiadas, pudiendo establecerse una relación de causa-efecto.

Un total de 212 de 218 pacientes $(97,2 \%)$ fueron valorables para seguridad (64,0\% HI y 36,0\% HS).

Las Reacciones Adversas (RA) más frecuentes fueron los trastornos generales de todo el organismo en un $7,1 \%$ de los casos $(n=15)$, los trastornos gastrointestinales en un $5,2 \%$ de los casos $(n=11)$, y los trastornos músculo-esqueléticos en un 3,3\% de los casos (n=7) (Tabla 6).

En una encuesta de satisfacción realizada al finalizar el tratamiento, el 66,3\% refirió estar "muy satisfecho", un 22,4\% "moderadamente satisfecho" y un 11,2\% "nada satisfecho" (Fig. 8). Analizados 


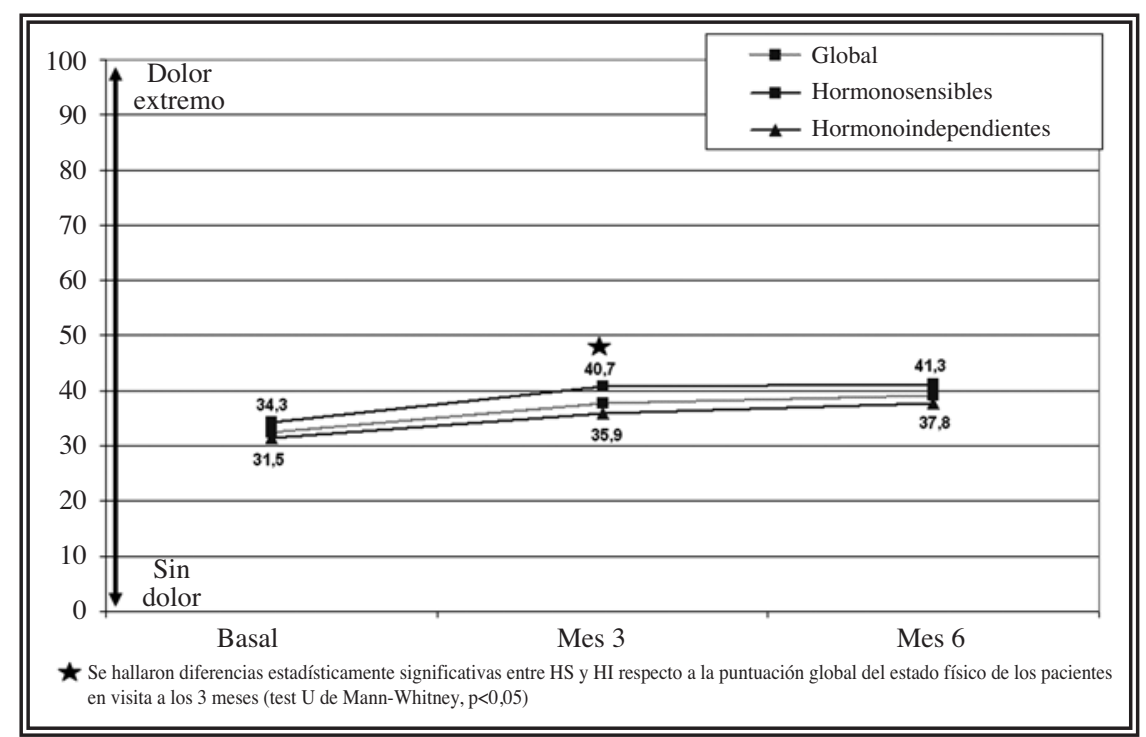

FIGURA 4. Evolución de la calidad de vida según puntuación global del estado fisico (SF-36).



FIGURA 5. Evolución de la calidad de vida según puntuación global del estado mental (SF-36).

por grupos, los resultados de las encuestas fueron similares, lo que tiene una especial significación clínica en los pacientes $\mathrm{HI}$ por el estado de multitratamiento oncológico en que se encuentran (análogos LH-RH, antiandrógenos, quimioterapia, etc.).

\section{DISCUSIÓN}

Las metástasis óseas son la mayor causa de morbilidad en cualquier tipo de cáncer. Las metástasis óseas están asociadas al dolor y a los eventos relacionados con el esqueleto. El objetivo principal de cualquier tratamiento es disminuir el dolor ${ }^{10}$, prevenir los eventos relacionados con el esqueleto $^{11} \mathrm{y}$ mantener la calidad de vida de los pacientes ${ }^{12}$.

En 2002, tras conocerse la alta potencia in vitro del ácido zoledrónico en la inhibición de la actividad osteolítica, y demostrar su efectividad en humanos en el retraso de la osteolisis en las metastasis óseas producidas por tumores sólidos, Saad et al. ${ }^{7}$ llevaron a cabo un estudio randomizado, controlado con placebo, en el que el ácido zoledrónico demostró un modesto y consistente efecto en el alivio del dolor. Esto acompañado con una significativa reducción de los eventos esqueléticos así como un retraso en su aparición, en el grupo tratado respecto al grupo placebo. Una de las principales limitaciones de este ensayo clínico fue que inicialmente no estaba diseñado para valorar el efecto analgésico del fármaco en estudio, y que la población incluida estaba restringida a pacientes en fase $\mathrm{HI}^{13}$. El estudio ha sido muy criticado por incluir solamente pacientes con enfermedad progresiva hormonoindependiente.

Nuestros resultados complementan los obtenidos en el ensayo clínico anterior, ya que esta diseñado para evaluar el alivio del dolor como una de las variables principales. Nuestros datos sugieren que el ácido zoledrónico consigue un alivio significativo del dolor (como se midió cuantitativamente empleando la escala EVA en movimiento y reposo), así como una mejoría en la movilidad; resultados notables en el primer mes de tratamiento y que se mantienen durante todo el estudio.

Además, un valor añadido de nuestro estudio es que no se limita a un subgrupo de pacientes, sino que se incluyeron todos los pacientes con enfermedad metastásica ósea, Independientemente de la 


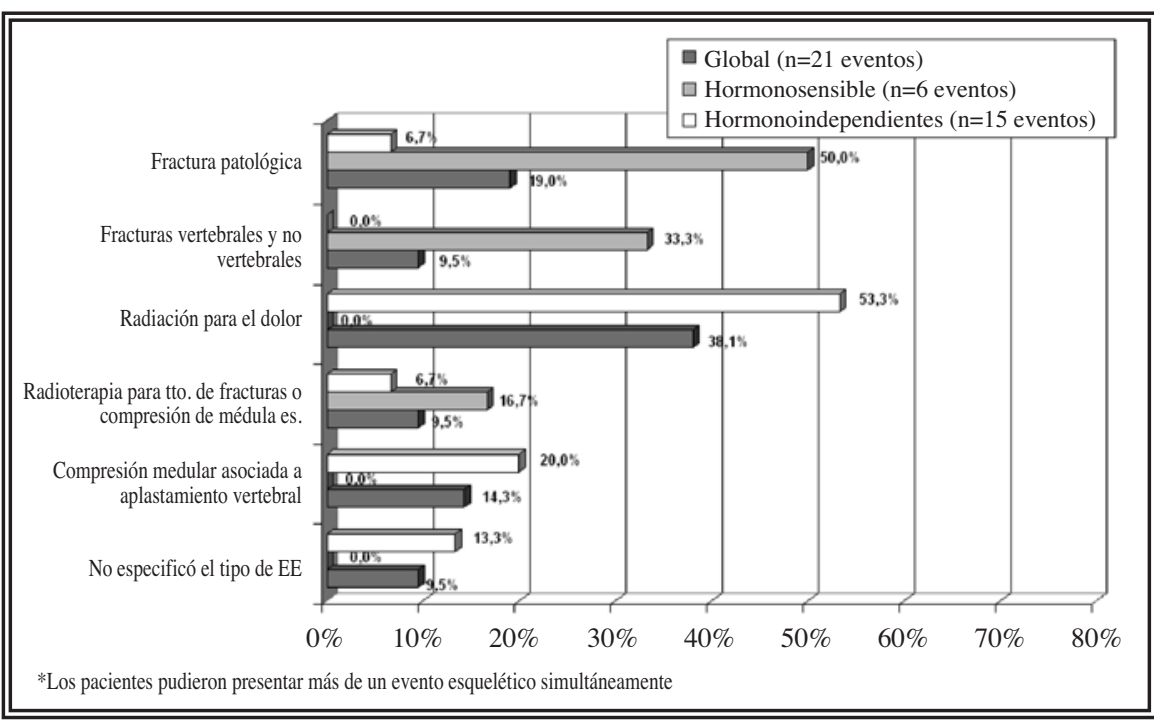

FIGURA 6. Tipo de Evento Esquelético.

En todos los pacientes tratados existió una mejoría del dolor en reposo y con el movimiento. $\mathrm{Si}$ bien dicha mejoría fue significativamente mayor en los pacientes HS a partir del tercer mes de tratamiento. Por tanto, estaría justificada la utilización precoz del ácido zoledrónico en pacientes con metástasis óseas sintomáticas en fase HS, puesto que el beneficio será mayor y mantenido en el tiempo (Figs. 1, 2 y 3). Como resultado de esto, la calidad de vida mejoró en todos los pacientes, especialmente en los HS, que una vez más obtuvieron una evolución de la calidad de la

fase HI o HS; por ser la población diana que acude vida más favorable que los HI.

El cuestionario SF-36 fue seleccionado por su a nuestras consultas y en los que nos planteamos si este fármaco aportaría un beneficio que justificase su uso. Al respecto, los datos obtenidos son concluyentes en las variables de interés estudiadas. alta sensibilidad y especificidad para evaluar el estado funcional físico y mental de los pacientes. Resultó de gran utilidad para objetivar la mejora del

Tabla 3.Tratamiento pautado para los Eventos Esqueléticos

\begin{tabular}{|c|c|c|c|c|c|c|c|c|c|}
\hline \multirow[b]{2}{*}{ a } & \multicolumn{3}{|c|}{ Global } & \multicolumn{3}{|c|}{ Hormonosensibles } & \multicolumn{3}{|c|}{ Hormonoindependientes } \\
\hline & $\mathbf{n}$ & $\%^{(1)}$ & $\%^{(2)}$ & $\mathbf{n}$ & $\%^{(1)}$ & $\%^{(2)}$ & $\mathbf{n}$ & $\%^{(1)}$ & $\%^{(2)}$ \\
\hline Pacientes con algún $\mathrm{EE}$ & 19 & $100 \%$ & - & 6 & $100 \%$ & - & 13 & $100 \%$ & - \\
\hline Sin tratamiento para el EE & 3 & $15,8 \%$ & - & $\mathbf{1}$ & $16,7 \%$ & - & 2 & $15,4 \%$ & - \\
\hline Con tratamiento para el $\mathrm{EE}^{(3)}$ & 16 & $84,2 \%$ & $100 \%$ & 5 & $83,3 \%$ & $100 \%$ & 11 & $84,6 \%$ & $100 \%$ \\
\hline Cirugía & 3 & $15,8 \%$ & $18,8 \%$ & 3 & $50,0 \%$ & $60,0 \%$ & 0 & $0,0 \%$ & $0,0 \%$ \\
\hline Fármacos & 3 & $15,8 \%$ & $18,8 \%$ & 1 & $16,7 \%$ & $20,0 \%$ & 2 & $15,4 \%$ & $18,2 \%$ \\
\hline Radioterapia & 12 & $63,2 \%$ & $75,0 \%$ & 1 & $16,7 \%$ & $20,0 \%$ & 11 & $84,6 \%$ & $100,0 \%$ \\
\hline
\end{tabular}

(1) Porcentaje calculado sobre el total de pacientes con algún Evento Esquelético.

(2) Porcentaje calculado sobre el total de pacientes con tratamiento para el Evento esquelético.

(3) Los pacientes pudieron indicar más de un tratamiento simultáneamente.

Tabla 4. Tiempo hasta la aparición del Evento Esquelético (meses)

\begin{tabular}{|c|c|c|c|c|}
\hline & $\begin{array}{l}\text { Tiempo hasta } \\
\text { (meses) }\end{array}$ & Esquelético & SD & IC(95\%) \\
\hline \multirow[t]{2}{*}{ Global } & Media & 10,65 & 0,29 & $10,09-11,22$ \\
\hline & Mediana $^{(1)}$ & - & - & - \\
\hline \multirow[t]{2}{*}{ Hormonosensibles ${ }^{(2)}$} & Media & 10,90 & 0,43 & $10,06-11,73$ \\
\hline & Mediana $^{(1)}$ & - & - & - \\
\hline \multirow[t]{2}{*}{ Hormonoindependientes ${ }^{(2)}$} & Media & 10,65 & 0,29 & $9,78-11,26$ \\
\hline & Mediana $^{(1)}$ & - & - & - \\
\hline
\end{tabular}

(1) No se pudo calcular la mediana del tiempo hasta la aparición del EE al no presentar el 50\% de los pacientes algún EE.

(2) No se hallaron diferencias estadísticamente significativas respecto a la distribución del tiempo hasta el Evento Esquelético entre hormonoindependientes y hormonosensibles (test Log Rank, $\mathrm{p}=0,561$; test Dreslow, $\mathrm{p}=0,430$ ). 


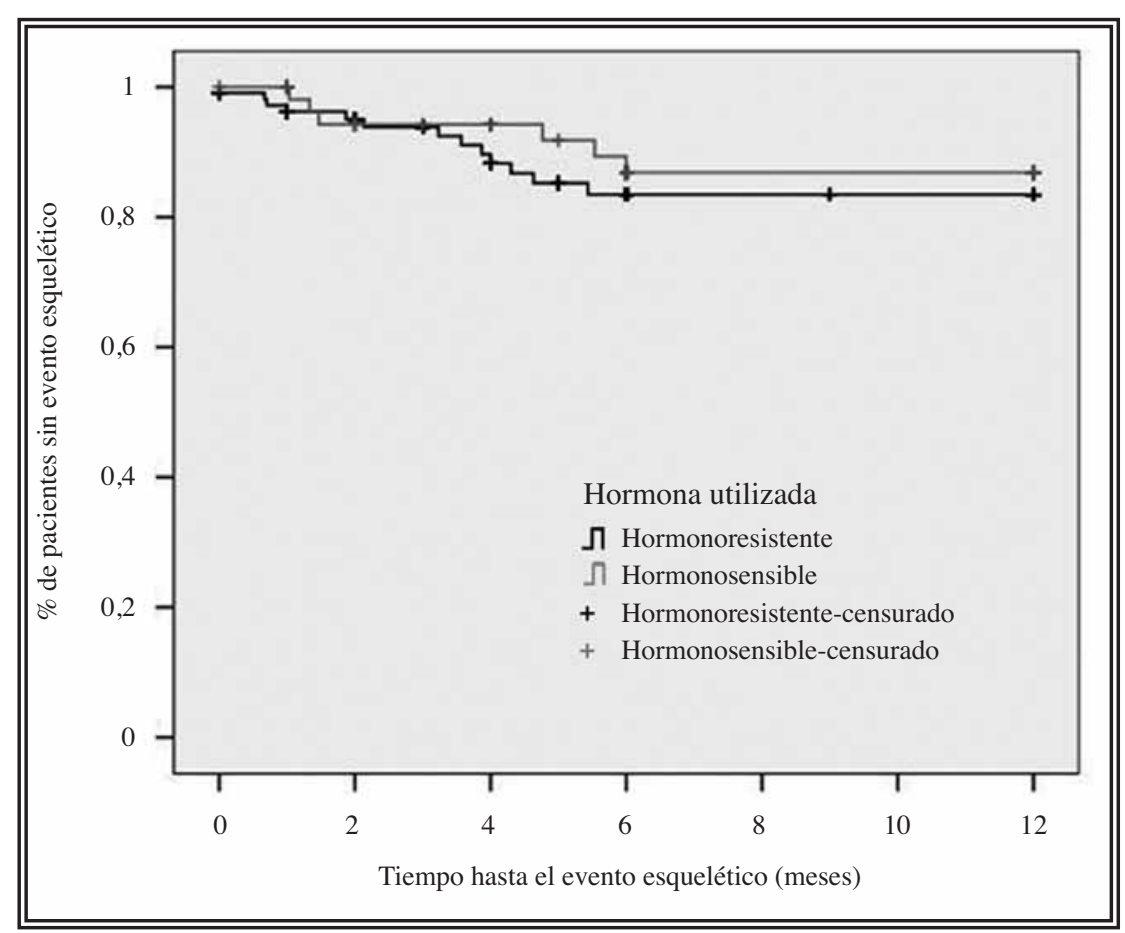

FIGURA 7. Curvas Kaplan-Meier para el TEE.

estado de ánimo físico y psicológico de los pacientes, como consecuencia de una percepción de que una mejoría del dolor suponía una mejoría de su proceso oncológico, lo que les permitía realizar una vida mas normal, con su quehacer diario menos alterado, mas horas de sueño y en definitiva una aceptación mejor de su status oncológico.

Un aspecto a considerar es la situación de partida que queremos revertir (de dolor, de deterioro de calidad de vida, de estado físico y/o mental), y que fue sensiblemente peor en los pacientes HI con respecto a los HS. Este hecho puede ser determinante en la respuesta que se obtuvo en cada grupo. Sabido es por los especialistas en Dolor, que este es mas difícil de revertir cuanto mayor es su intensidad, precisando de manipulaciones cada vez mas agresivas y complejas cuanto mas se ha dejado evolucionar. De forma similar podríamos razonar en el cáncer de próstata con enfermedad ósea metastásica: en la fase precoz de la afectación, que suele ser la etapa HS, la destrucción ósea por osteolisis debe ser necesariamente menor que en etapas tardías de la enfermedad metastásica, que suele ser la de progresión HI. Por tanto, en principio resultaría más fácil detener la fisiopatología ósea en su etapa incipiente que en la progresiva HI. Dicho fenómeno estudiado con el ácido zoledrónico ha sido bien documentado a nivel de su mecanismo de acción ${ }^{14,15}$.

En nuestro estudio hemos demostrado como el ácido zoledrónico retrasa la osteolisis. El resultado fue un descenso significativo del marcador de resorción ósea, como fue el N-telopéptido, en todos los pacientes, tanto HS como HI. Asociado a este dato, observamos una disminución significativa del dolor óseo en todos los pacientes, tanto HS como HI. Si bien el círculo vicioso de destrucción ósea iniciado con la metástasis, puede ser más sensible a la acción del fármaco cuanto antes actúe este, con respecto a los casos en que por una larga evolución el hueso este muy afectado y sea más resistente a su detención por el bisfosfonato. Todo ello abogaría por una utilización precoz del bisfosfonato con un mayor beneficio en hueso menos dañado y con dolor óseo menos intenso.

Tabla 5. Bioquímica: Fosfatasa Alcalina ósea y NTX

\begin{tabular}{|c|c|c|c|c|c|c|c|c|c|c|c|c|c|}
\hline & & \multicolumn{3}{|c|}{ Global } & \multicolumn{5}{|c|}{ Hormonosensibles } & \multicolumn{4}{|c|}{ Hormonoindependientes } \\
\hline & & $\mathbf{n}^{(1)}$ & Media & SD & $\mathbf{P}^{(2)}$ & $\mathbf{n}^{(1)}$ & Media & SD & $\mathbf{P}^{(2)}$ & $\mathbf{n}^{(1)}$ & Media & SD & $\mathbf{P}^{(2)}$ \\
\hline \multirow[t]{2}{*}{$\begin{array}{l}\text { Fosfatasa alcalina } \\
\text { ósea (ng/L) }\end{array}$} & Basal & 113 & 591,4 & 1452,7 & 0,1789 & 34 & 310,5 & 467,7 & 0,0840 & 79 & 712,2 & 1699,6 & 0,4926 \\
\hline & Mes 6 & 57 & 329,6 & 506,5 & & 25 & 144,6 & 194,1 & & 32 & 474,2 & 620,6 & \\
\hline \multirow[t]{2}{*}{$\begin{array}{l}\text { NTX (nmol BCE/ } \\
\left.\text { mmol creat }^{(3)}\right)\end{array}$} & Basal & 143 & 171,7 & 204,2 & $<0,0001$ & 50 & 142,5 & 188,4 & 0,0001 & 93 & 187,3 & 211,5 & 0,0001 \\
\hline & Mes 6 & 54 & 45,2 & 61,4 & & 26 & 38,8 & 58,0 & & 28 & 51,2 & 64,9 & \\
\hline
\end{tabular}

(1): En algunos casos no se especificó el dato.

(2): Test de Wilcoxon.

(3): BCE: bone colagen equivalents. 
Tabla 6. Reacciones Adversas

\begin{tabular}{|c|c|c|c|c|c|c|c|c|c|}
\hline TIPO & NR & NP & $\%$ & NR & NP & $\%$ & NR & NP & $\%$ \\
\hline \multicolumn{10}{|l|}{ Trastornos del sistema } \\
\hline Artralgia & 3 & 3 & $1,4 \%$ & 1 & 1 & $0,5 \%$ & 2 & 2 & $0,9 \%$ \\
\hline Mialgia & 2 & 2 & $0,9 \%$ & 1 & 1 & $0,5 \%$ & 0 & 0 & $0,0 \%$ \\
\hline Dolor Esquelético & 2 & 2 & $0,9 \%$ & 1 & 1 & $0,5 \%$ & 1 & 1 & $0,5 \%$ \\
\hline \multicolumn{10}{|c|}{ Trastornos del sistema nervioso } \\
\hline Mareo & 3 & 3 & $1,4 \%$ & 1 & 1 & $0,5 \%$ & 2 & 2 & $0,9 \%$ \\
\hline Neuralgia & 1 & 1 & $0,5 \%$ & 0 & 0 & $0,0 \%$ & 1 & 1 & $0,5 \%$ \\
\hline \multicolumn{10}{|l|}{ Trastornos del sistema } \\
\hline Estreñimiento & 4 & 4 & $1,9 \%$ & 1 & 1 & $0,5 \%$ & 3 & 3 & $1,4 \%$ \\
\hline Diarrea & 1 & 1 & $0,5 \%$ & 1 & 1 & $0,5 \%$ & 0 & 0 & $0,0 \%$ \\
\hline Vómitos & 5 & 5 & $2,4 \%$ & 4 & 4 & $1,9 \%$ & 0 & 0 & $0,0 \%$ \\
\hline Nauseas & 4 & 4 & $1,9 \%$ & 2 & 2 & $0,9 \%$ & 1 & 1 & $0,5 \%$ \\
\hline \multicolumn{10}{|c|}{ Trastornos generales de todo } \\
\hline Edemas & 3 & 3 & $1,4 \%$ & 2 & 2 & $0,9 \%$ & 1 & 1 & $0,5 \%$ \\
\hline Astenia & 2 & 2 & $0,9 \%$ & 2 & 2 & $0,9 \%$ & 0 & 0 & $0,0 \%$ \\
\hline Fiebre/escalofríos & 5 & 5 & $2,4 \%$ & 3 & 3 & $1,4 \%$ & 2 & 2 & $0,9 \%$ \\
\hline Malestar General & 2 & 2 & $0,9 \%$ & 1 & 1 & $0,5 \%$ & 1 & 1 & $0,5 \%$ \\
\hline Dolor general & 3 & 3 & $1,4 \%$ & 1 & 1 & $0,5 \%$ & 2 & 2 & $0,9 \%$ \\
\hline Síntomas Tipo Gripe & 4 & 4 & $1,9 \%$ & 0 & 0 & $0,0 \%$ & 4 & 4 & $1,9 \%$ \\
\hline
\end{tabular}

NR: $\mathrm{n}^{\circ}$ de efectos adversos;

NP: $\mathrm{n}^{\circ}$ de pacientes con el efecto adverso (hay pacientes que se contabilizan más de una vez, por tener varias reacciones adversas); \%: porcentaje de pacientes con el efecto adverso sobre el total de pacientes valorables por seguridad.



FIGURA 8. Satisfacción con el tratamiento al finalizar.

Respecto al tiempo de aparición de los eventos relacionados con el esqueleto, no tenemos conclusiones ya que no tenemos un grupo control o de placebo con el que comparar si existe un retraso significativo en su aparición y número. Tan sólo podemos considerar que los hallazgos son compatibles con los descritos en otros estudios realizados y comunicados en la literatura, en los que el ácido zoledrónico demuestra una acción preventiva en la aparición de eventos relacionados con el esqueleto $^{3,7,16,17}$.

Un efecto no medido en nuestro estudio, ha sido el no evaluar la acción del fármaco sobre la densidad ósea. El trabajo de Smith et al. ${ }^{18}$ demostró que tras la deprivación androgénica realizada en estos pacientes, existe una disminución de la densidad ósea, minimizada por el ácido zoledrónico. Nuestros resultados, de haberlos cuantificado mediante densitometría, estarian en coherencia con estos resultados comunicados por Smith et al., y sería una variable más a tener en cuenta a la hora de sopesar los beneficios/riesgos de utilizar este fármaco. 
Para concluir, queremos nuevamente resaltar que éste es un estudio prospectivo observacional, abierto, multicéntrico, no controlado, en pacientes con enfermedad metastásica por cáncer prostático. El objetivo principal era evaluar la efectividad en alivio del dolor óseo en reposo y en movimiento, así como cuantificar la incidencia y el tiempo de aparición de eventos esqueléticos. Globalmente, este estudio demuestra un beneficio significativo en todas estas variables con la utilización del ácido zoledrónico, lo que se reflejó en una mejoría del estado funcional y de la calidad de vida de estos pacientes.

A diferencia de todos los estudios publicados hasta la fecha, que sólo reclutaron pacientes en fase de progresión HI, hemos incluido pacientes con enfermedad metastásica independientemente de la fase terapéutica, HS o HI, por ser el tipo de pacientes que habitualmente acuden a nuestra consulta externa. Hemos comparado la efectividad/tolerabilidad del tratamiento con ácido zoledrónico en ambos grupos, llegando a la conclusión de que ambos grupos de pacientes se benefician del tratamiento, en todas las variables de efectividad evaluadas. La incidencia de eventos esqueléticos fue baja y su aparición tardía. Y todo ello con una toxicidad mínima, no relevante clínicamente, y con un grado de satisfacción elevado por parte de la mayoría de los pacientes (66\%).

Nuestros resultados, por tanto, son extrapolables a todos los pacientes que habitualmente tratamos en nuestra consulta con un cáncer de próstata metastático, para los que deberemos considerar que el tratamiento oncológico especifico se debe complementar con el tratamiento de la enfermedad ósea mediante fármacos como el ácido zoledrónico, buscando el mayor beneficio terapéutico posible.

\section{REFERENCIAS}

1. Guarneri V, Donati S, Nicolini M, Giovannelli S, D’Amico R, Conte PF. Renal safety and efficacy of i.v. bisphosphonates in patients with skeletal metastases treated for up to 10 Years. Oncologist. 2005;10(10):842-848.

2. Wellington K, Goa KL. Zoledronic acid: a review of its use in the management of bone metastases and hypercalcaemia of malignancy. Drugs. 2003;63(4):417-437.

3. Santini D, Vincenzi B, Tonini G, Scarpa S, Baldi A. Zoledronic acid exhibits inhibitory effects on osteoblastic and osteolytic metastases of prostate cancer. Clin Cancer Res. 2003;9(8):32153216 .
4. Coleman RE, Seaman JJ. The role of zoledronic acid in cancer: clinical studies in the treatment and prevention of bone metastases. Semin Oncol. 2001;28(Suppl 6):11-16.

5. Coleman RE. Future directions in the treatment and prevention of bone metastases. Am J Clin Oncol. 2002;(Suppl 1):3238.

6. Fulfaro F, Leto G, Badalamenti G, Arcara C, Cicero G, Valerio $\mathrm{MR}$, et al. The use of zoledronic acid in patients with bone metastases from prostate carcinoma: effect on analgesic response and bone metabolism biomarkers. J Chemother. 2005; 17(5): 555-559.

7. Saad F, Gleason DM, Murray R, Tchekmedyian S, Venner P, Lacombe L, et al. A Randomized, Placebo-Controlled Trial of Zoledronic Acid in Patients with Hormone-Refractary Metastatic Prostate Carcinoma. J Natl Cancer Inst. 2002;94(19): 1458-1468.

8. Saad F, McKiernan J, Eastham J. Rationale for zoledronic acid therapy in men with hormone-sensitive prostate cancer with or without bone metastasis. Urol Oncol. 2006;24(1):4-12.

9. Polascik TJ, Given RW, Metzger C, Julian SR, Vestal JC, Karlin GS, et al. Open-label trial evaluating the safety and efficacy of zoledronic acid in preventing bone loss in patients with hormone-sensitive prostate cancer and bone metastases. Urology. 2005;66(5):1054-1059.

10. Cózar JM, Galvez R, Tallada M. Modalidades terapeuticas del dolor oseo en el cáncer de prostata. Actas Urol Esp. 1999;23 (5):464-470.

11. Heidenreich A. Therapy of hormone-refractory prostate cancer. Urologe. 2005;44(12):1481-1494.

12. Weinfurt KP, Li Y, Castel LD, Saad F, Timbie JW, Glendenning GA, et al. The significance of skeletal-related events for the health-related quality of life of patients with metastatic prostate cancer. Ann Oncol. 2005;16(4):579-584.

13. Saad F, Gleason DM, Murray R, Tchekmedyian S, Venner P, Lacombe L, et al. Long-term efficacy of zoledronic acid for the prevention of skeletal complications in patients with metastatic hormone-refractory prostate cancer. J Natl Cancer Inst. 2004;96(11):879-882.

14. Corey E, Brown LG, Quinn JE, Poot M, Roudier MP, Higano CS, et al. Zoledronic acid exhibits inhibitory effects on osteoblastic and osteolytic metastases of prostate cancer. Clin Cancer Res. 2003;9(1):295-306.

15. Smith MR. Osteoclast-targeted therapy for prostate cancer. Curr Treat Options Oncol. 2004;5(5):367-375.

16. Saad F. Clinical benefit of zoledronic acid for the prevention of skeletal complications in advanced prostate cancer. Clin Prostate Cancer. 2005;4(1):31-37.

17. Gilbert SM, Olsson CA, Benson MC, McKiernan JM. The role of intravenous zoledronic acid in the management of high-risk prostate cancer. Curr Opin Urol. 2003;13(2):133-135.

18. Smith MR. The role of bisphosphonates in men with prostate cancer receiving androgen deprivation therapy. Oncology. 2004;18(Suppl 3):21-25.

Correpondencia autor: Dr. J.M. Cózar Olmo

Servicio de Urología. Hospital Universitario Virgen de las Nieves.

Avda. Fuerzas Armadas, nº - 18014 Granada

Tel: 686873340/Fax: 958020145

E-mail autor: cozarjm@yahoo.es

Información artículo: Original - Cáncer de próstata

Trabajo recibido: marzo 2007

Trabajo aceptado: marzo 2007 